Article

\title{
Boron Monochalcogenides; Stable and Strong Two-Dimensional Wide Band-Gap Semiconductors
}

\author{
Bohayra Mortazavi ${ }^{1,2, *(1)}$ and Timon Rabczuk ${ }^{3}$ \\ 1 Institute of Structural Mechanics, Bauhaus-Universität Weimar, Marienstr. 15, D-99423 Weimar, Germany \\ 2 Institute for Materials Science and Max Bergman Center of Biomaterials, TU Dresden, \\ 01062 Dresden, Germany \\ 3 College of Civil Engineering, Department of Geotechnical Engineering, Tongji University, Shanghai 200092, \\ China; timon.rabczuk@uni-weimar.de \\ * Correspondence: bohayra.mortazavi@gmail.com; Tel.: +49-157-8037-8770; Fax: +49-364-358-4511
}

Received: 13 May 2018; Accepted: 13 June 2018; Published: 15 June 2018

\begin{abstract}
In this short communication, we conducted first-principles calculations to explore the stability of boron monochalcogenides ( $B X, X=S$, Se or Te), as a new class of two-dimensional (2D) materials. We predicted BX monolayers with two different atomic stacking sequences of ABBA and $\mathrm{ABBC}$, referred in this work to $2 \mathrm{H}$ and $1 \mathrm{~T}$, respectively. Analysis of phonon dispersions confirm the dynamical stability of $\mathrm{BX}$ nanosheets with both $2 \mathrm{H}$ and $1 \mathrm{~T}$ atomic lattices. Ab initio molecular dynamics simulations reveal the outstanding thermal stability of all predicted monolayers at high temperatures over $1500 \mathrm{~K}$. BX structures were found to exhibit high elastic modulus and tensile strengths. It was found that BS and BTe nanosheets can show high stretchability, comparable to that of graphene. It was found that all predicted monolayers exhibit semiconducting electronic character, in which $2 \mathrm{H}$ structures present lower band gaps as compared with $1 \mathrm{~T}$ lattices. The band-gap values were found to decrease from BS to BTe. According to the HSE06 results, 1T-BS and 2H-BTe show, respectively, the maximum $(4.0 \mathrm{eV})$ and minimum $(2.06 \mathrm{eV})$ electronic band gaps. This investigation introduces boron monochalcogenides as a class of 2D semiconductors with remarkable thermal, dynamical, and mechanical stability.
\end{abstract}

Keywords: two-dimensional semiconductor; first-principles; mechanical; thermal

\section{Introduction}

After the great success of graphene [1-3], during the last decade the interest in two-dimensional (2D) materials has been continuously increased. One of the critical factors that has contributed largely in astonishing advances in the field of 2D materials is the available advanced theoretical techniques that enabled researchers to examine the stability of new compositions and estimate properties and suggest possible synthesis routes [4-7] using pure computational experiments. As an example, in 2015, successful experimental realization of borophene nanosheets were reported through epitaxial growth of boron atoms on a Ag surface [8,9]. Nevertheless, prior to these experimental successes, stability, fabrication conditions, and electronic properties of various borophene structures were theoretically predicted $[10,11]$. Currently, the family of $2 \mathrm{D}$ materials includes prominent members, such as: hexagonal boron-nitride [12,13], carbon-nitride 2D networks [14-17], silicene [18,19], germanene [20], transition metal dichalcogenides [21-23], phosphorene [24,25], and indium monochalcogenides [26].

In the most recent theoretical and experimental work by Cherednichenko et al. [27], the phase transition in a rhombohedral boron monosulfide ( $r$-BS) bulk layered structure was studied. Interestingly, the atomic structure of $r$-BS in the single-layer form is similar to that of indium selenide, which was recently synthesized in 2D forms by Bandurin et al. [26]. Worthy noting is that the stability of 
single-layer indium chalcogenides were first confirmed theoretically by Zólyomi and co-workers [28], a few years earlier than the experimental realization [26]. Also noteworthy is that BS monolayers with four different atomic lattices were most recently theoretically predicted by Fan and co-workers [29]. Motivated by the previous investigations [26,28], in this work we decided to examine the stability of single-layer boron monochalcogenides, with a chemical formula of BX, where $\mathrm{X}$ stands for S, Se, or Te chalcogen elements. To this aim, we conducted density functional theory calculations to explore the structural and thermal stability, and the basic mechanical and electronic properties of single-layer boron monochalcogenides as a class of 2D materials.

\section{Computational Method}

First-principles density functional theory (DFT) simulations in this work were conducted using the Vienna ab initio simulation package (VASP) [30-32]. The generalized gradient approximation (GGA) exchange-correlation functional of Perdew-Burke-Ernzerhof (PBE) [33] and plane wave basis set with an energy cut-off of $500 \mathrm{eV}$ were also employed. The conjugate gradient method within the tetrahedron method with Blöchl corrections [34] was used for the geometry optimizations, with termination criteria of $10^{-5} \mathrm{eV}$ and $0.005 \mathrm{eV} / \AA$ for the energy and forces, respectively, using a $19 \times 19 \times 1$ Monkhorst-Pack [35] k-point mesh size. Since the PBE functional underestimates the band gap values, we used Heyd-Scuseria-Ernzerhof (HSE06) [36] method to report the electronic band gaps of predicted monolayers. To assess the dynamical stability of BX monolayers, we conducted density functional perturbation theory (DFPT) simulations for $4 \times 4$ super-cells within the finite displacement method using the Phonopy package [37]. The thermal stability of single-layer BX was explored via ab initio molecular dynamics (AIMD) simulations for $3 \times 3$ super-cells with a time increment of $1 \mathrm{fs}$ and $2 \times 2 \times 1$ k-point mesh size. To evaluate the mechanical properties, we conducted uniaxial tensile modelling for the unit-cells. To this aim, we increased the size of the periodic simulation box along the loading direction with a constant engineering strain step. In order to observe the uniaxial stress condition, the simulation box size along the transverse direction of the loading was changed to reach negligible stress along this direction.

\section{Results and Discussions}

Like indium chalcogenides [28], predicted BX nanomembranes in this work also show hexagonal lattices, in which two layers of columned B atoms are sandwiched between two chalcogen atom layers. Depending on the arrangement of chalcogen atoms, BX nanosheets can exhibit two different atomic structures. In accordance with transition metal dichalcogenides' atomic lattices [38-40], we call the graphene-like BX with an atomic stacking sequence of ABBA as the $2 \mathrm{H}$ structure. On the other hand, $\mathrm{BX}$ nanosheets with a $1 \mathrm{~T}$ phase present an atomic stacking sequence of $\mathrm{ABBC}$, in which the chalcogen atoms, either on the bottom or top, are placed in the hollow center of the hexagonal lattice. Figure 1, illustrates the atomic lattices of $2 \mathrm{H}$ - and 1T-BX monolayers. The atomic lattice of $\mathrm{BX}$ monolayers can be well defined by the hexagonal lattice constant and B-B and B-X bond lengths. The lattice parameters of energy minimized BX monolayers with hexagonal unit-cells are summarized in Table 1 . To compare the stability of $2 \mathrm{H}$ and $1 \mathrm{~T}$ phases, we calculated the energy per atom for $\mathrm{BX}$ monolayers and the results are also included in Table 1. Interestingly, boron monochalcogenides with 1T lattice show lower energies and, thus, are more stable in comparison with $2 \mathrm{H}$ counterparts. 

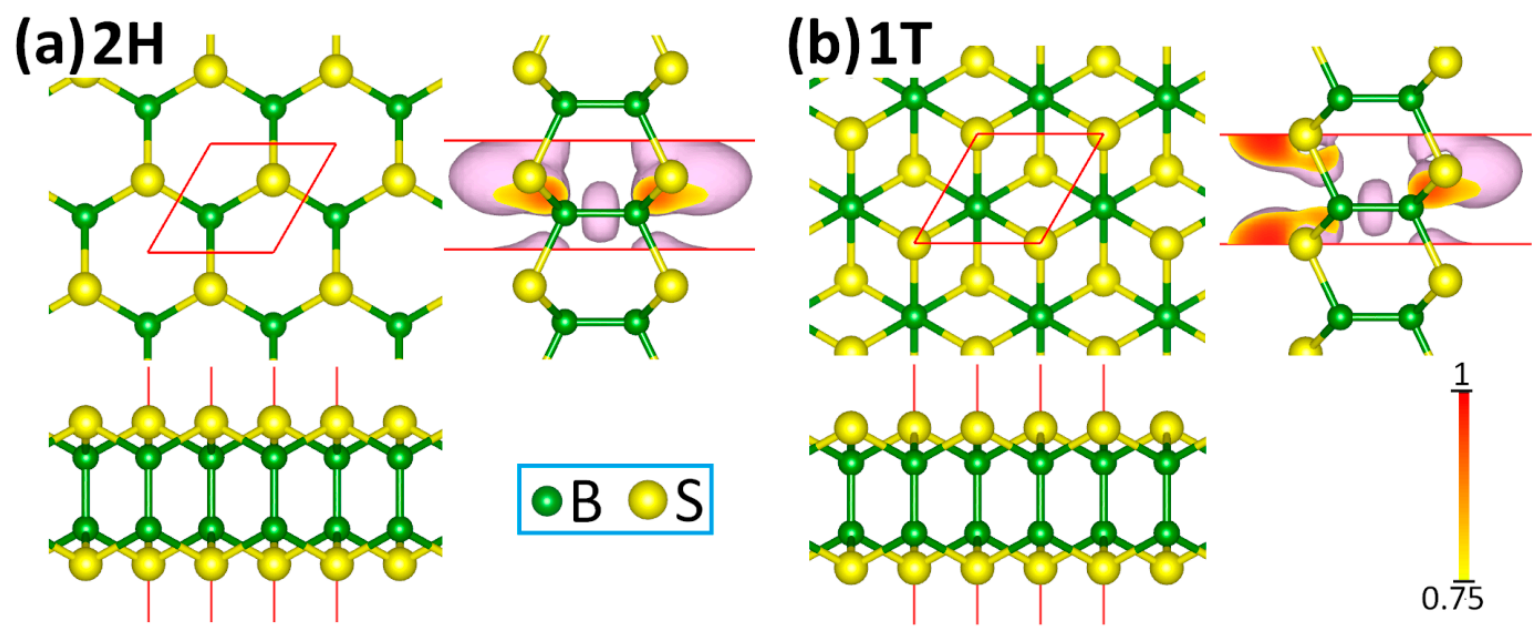

Figure 1. Top and side views of atomic configuration of single-layer BS with $2 \mathrm{H}$ and $1 \mathrm{~T}$ lattices, with $\mathrm{ABBA}$ and $\mathrm{ABBC}$ stacking sequences, respectively. For the right side views the electron localization function 3D profiles are also plotted. The red box shows the unit-cell which includes four atoms. The VESTA [41] package was employed to illustrate the atomic structures.

Table 1. Lattice parameters of energy minimized BX monolayers. Here, $L_{B-B}$ and $L_{B-X}, E_{\text {unit-cell }}$, and $\triangle \mathrm{Q}$ stand for B-B and B-X bond lengths, energy per atom of a unit-cell, and Bader results for charge transfer from a single $\mathrm{B}$ atom to a chalcogen atom, respectively.

\begin{tabular}{ccccccc}
\hline \multicolumn{2}{c}{ Structure } & Lattice Constant $(\AA)$ & $\boldsymbol{L}_{\boldsymbol{B}-\boldsymbol{B}}(\AA)$ & $\boldsymbol{L}_{\boldsymbol{B}-\boldsymbol{X}}(\AA)$ & $\boldsymbol{E}_{\text {unit-cell }}(\mathbf{e V})$ & $\Delta \mathbf{Q}$ \\
\hline \multirow{2}{*}{$\mathrm{BS}$} & $\mathrm{2H}$ & 3.041 & 1.728 & 1.950 & -5.813 & 1.88 \\
& $1 \mathrm{~T}$ & 3.056 & 1.704 & 1.954 & -5.818 & 1.86 \\
\hline \multirow{2}{*}{$\mathrm{BSe}$} & $2 \mathrm{H}$ & 3.259 & 1.712 & 2.087 & -5.241 & 0.68 \\
& $1 \mathrm{~T}$ & 3.274 & 1.686 & 2.087 & -5.256 & 0.58 \\
\hline \multirow{2}{*}{$\mathrm{BTe}$} & 2H & 3.565 & 1.713 & 2.314 & -4.720 & -0.1 \\
& $1 \mathrm{~T}$ & 3.589 & 1.681 & 2.316 & -4.746 & -0.11 \\
\hline
\end{tabular}

In order to better understand the bonding nature in boron monochalcogenide nanomembranes, we also plotted the electron localization function (ELF) [42] for BS monolayers in Figure 1. It is worth reminding the reader that ELF is a normalized and position-dependent function in which the values close to one corresponds to a high probability of finding electron localizations, and ELF equal to one half corresponds to the region of electron gas-like behaviour. We found that for the all boron monochalcogenides lattices, the ELF values around the centre of all bonds are greater than 0.75, confirming the covalent bonding nature in this class of 2D materials. As is clear in Figure 1, electron localizations also occur around the chalcogen atoms, which is due to their higher valance electrons. To investigate the charge transfer between B and chalcogen atoms, we conducted the Bader charge analysis [43] and the results for different atomic lattices are also included in Table 1. Interestingly, for BS and BSe monolayers, considerably higher electronegativity of S and Se atoms in comparison with B atoms lead to charge gain from $B$ atoms. These charge transfers accordingly induce ionic interactions between heteronuclear bonds in BS and BSe nanomembranes. In these cases, the charge transfer is higher for the $2 \mathrm{H}$ phase as compared with $1 \mathrm{~T}$ lattices, resulting in stiffer bonding and smaller hexagonal lattice constants for $2 \mathrm{H}$ lattices. For BTe nanosheets the Bader results, however, suggest that the charge transfer is convincingly negligible.

In order to analyse the dynamical stability of the single-layer BX lattices, the phonon dispersions along the high symmetry $\Gamma-\mathrm{M}-\mathrm{K}-\Gamma$ directions were computed and the obtained results are shown in Figure 2. As is clear for all the predicted monolayers, phonon dispersions are convincingly free of 
imaginary vibrational frequencies and thus they are all stable from dynamical point of view. For all the structures, in the phonon dispersions two gaps in frequencies are observable. For all the monolayers before the first gap in the phonons frequency, the chalcogen atoms contribute mostly to the vibrations. In contrast, boron atoms with lighter atomic weights were found to dominate the vibrations after the first gap in the phonon dispersions. By increasing the weight of the chalcogen atoms, the gaps in the phonon dispersions widen. For the predicted BX monolayers, 1T lattices were found to exhibit slightly higher frequencies in comparison with $2 \mathrm{H}$ phases.
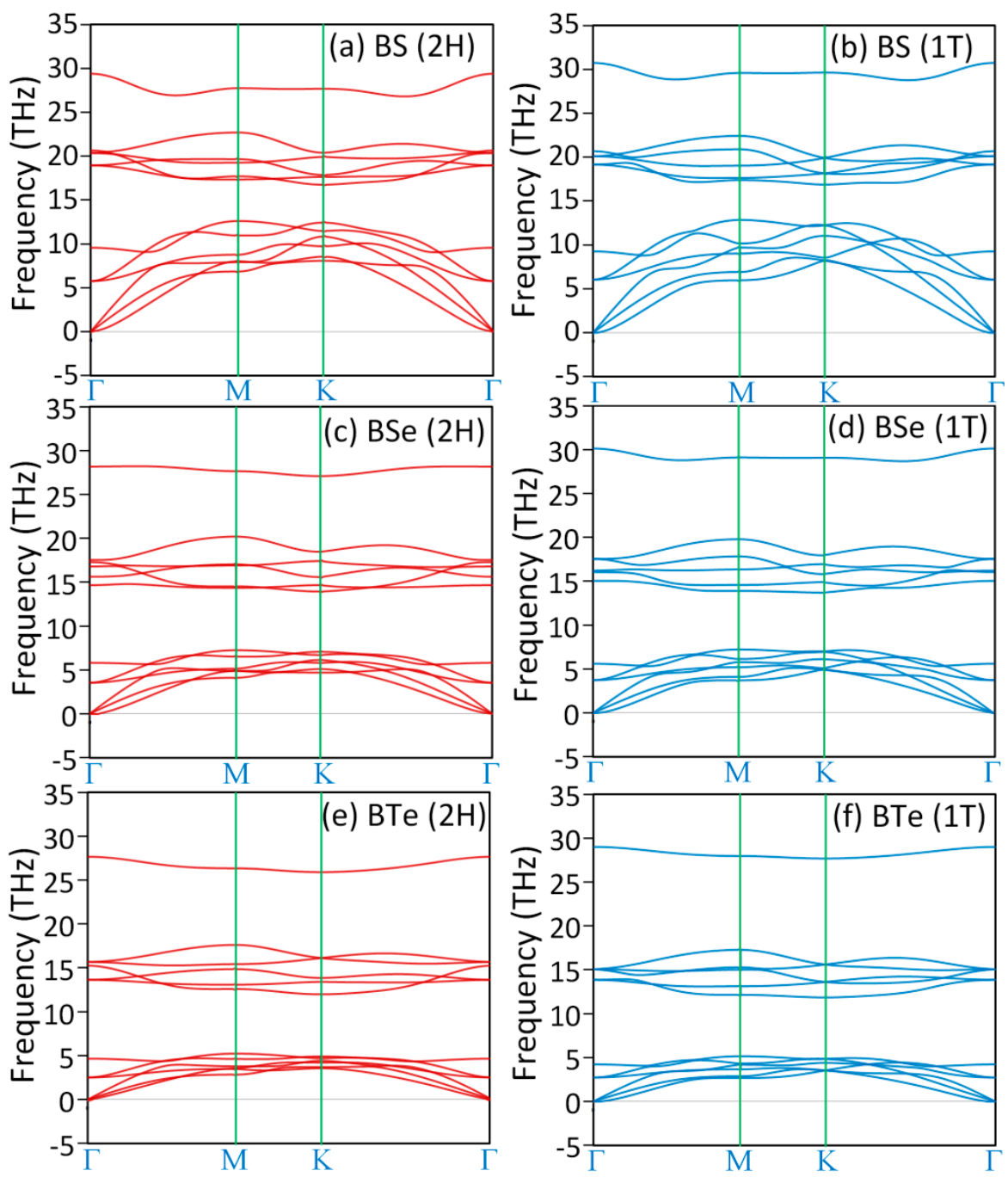

Figure 2. Phonon dispersions of free-standing and single-layer BX nanosheets.

Thermal stability at high temperatures is another critical property that is highly desirable for every material in engineering applications. We, therefore, next examined the thermal stability of single-layer boron monochalcogenides using the AIMD simulations at high temperatures. To this goal, AIMD calculations were conducted at different temperatures of $500 \mathrm{~K}, 1000 \mathrm{~K}, 1500 \mathrm{~K}$, and $2000 \mathrm{~K}$, for 15 ps long simulations. Snapshots of the BX monolayers with $2 \mathrm{H}$ and $1 \mathrm{~T}$ phases after the $15 \mathrm{ps}$ of AIMD simulations at high temperatures of $1500 \mathrm{~K}$ and $2000 \mathrm{~K}$ are illustrated in Figure 3. As a remarkable finding, all predicted BS and BSe monolayers were found to stay intact at the high temperature of $2000 \mathrm{~K}$. As is clear, nevertheless, BTe monolayers with the both $2 \mathrm{H}$ and 1T lattices were disintegrated at $2000 \mathrm{~K}$. The obtained results confirm the outstanding thermal stability of BX monolayers, which are all able to keep their structures intact at high temperatures, like $1500 \mathrm{~K}$. As it was discussed earlier, $1 \mathrm{~T}$ 
phases were found to be more energetically favourable since their lattice energies were slightly lower than those of $2 \mathrm{H}$ lattices. Higher energetic stability of $1 \mathrm{~T}$ phases suggest that they are more probable to be experimentally realized, which is in agreement with the $r$-BS bulk layered structure with the well-known 1T atomic lattice [27]. Our results shown in Figure 3, confirm no sign of $2 \mathrm{H}$ to $1 \mathrm{~T}$ phase transition at high temperatures, as $2 \mathrm{H}$ phases could keep their original hexagonal and graphene-like lattices intact. This finding not only reveals the remarkable thermal stability of BX nanomembranes with $1 \mathrm{~T}$ and $2 \mathrm{~T}$ phases, but also shows that the energy barriers for $2 \mathrm{H}$ to $1 \mathrm{~T}$ phase transition in $\mathrm{BX}$ monolayers are considerably high, which cannot be passed by high temperature annealing.

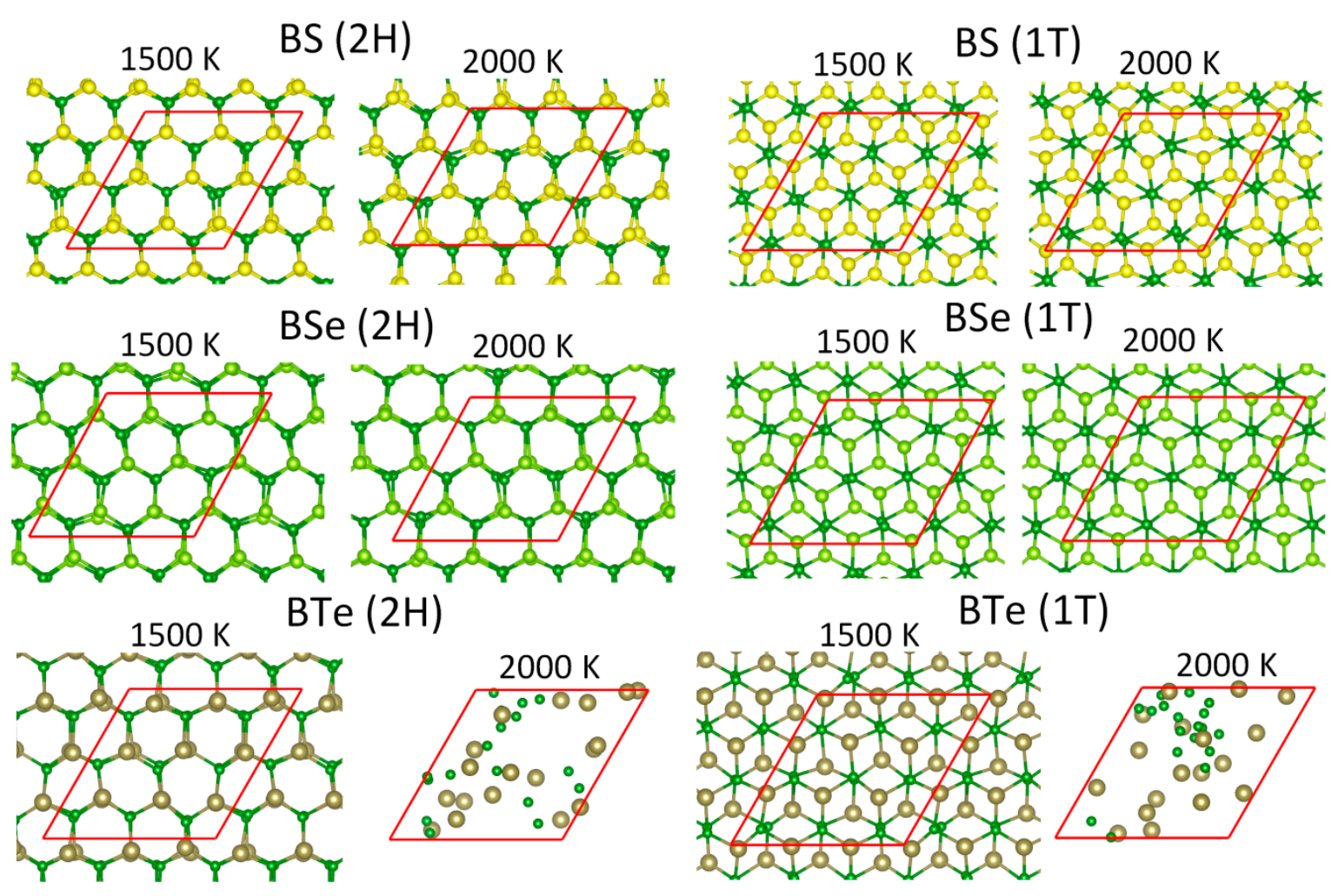

Figure 3. Snapshots of single-layer boron monochalcogenides at different temperatures of $1500 \mathrm{~K}$ and $2000 \mathrm{~K}$ after the AIMD simulations for 15 ps.

In Figure 4 band structures of BX monolayers along high symmetry directions predicted by PBE method are illustrated. Our results indicate that all boron monochalcogenides monolayers are wide and indirect band gap semiconductors. This observation suggests the limited application prospect of predicted monolayers as nanotransistors in post-silicon electronic. For the all BS monolayers valence band maximum (VBM) occurs around the $\Gamma$ point. For the single-layer boron monochalcogenides with 1T atomic lattice, conduction band minimum (CBM) happens around the M point. In the case of $B X$ monolayers with $2 \mathrm{H}$ atomic configuration, the $\mathrm{CBM}$, however, occurs at $\mathrm{M}$ and $\mathrm{K}$ points. The band gaps of BS, BSe, and BTe monolayers with a $2 \mathrm{H}$ lattice within the PBE functional were measured to be $2.8 \mathrm{eV}, 2.07 \mathrm{eV}$, and $1.49 \mathrm{eV}$, respectively. The corresponding values for 1T-BS, -BSe, and -BTe monolayers were found to be higher, $2.87 \mathrm{eV}, 2.5 \mathrm{eV}$, and $1.7 \mathrm{eV}$, respectively. 

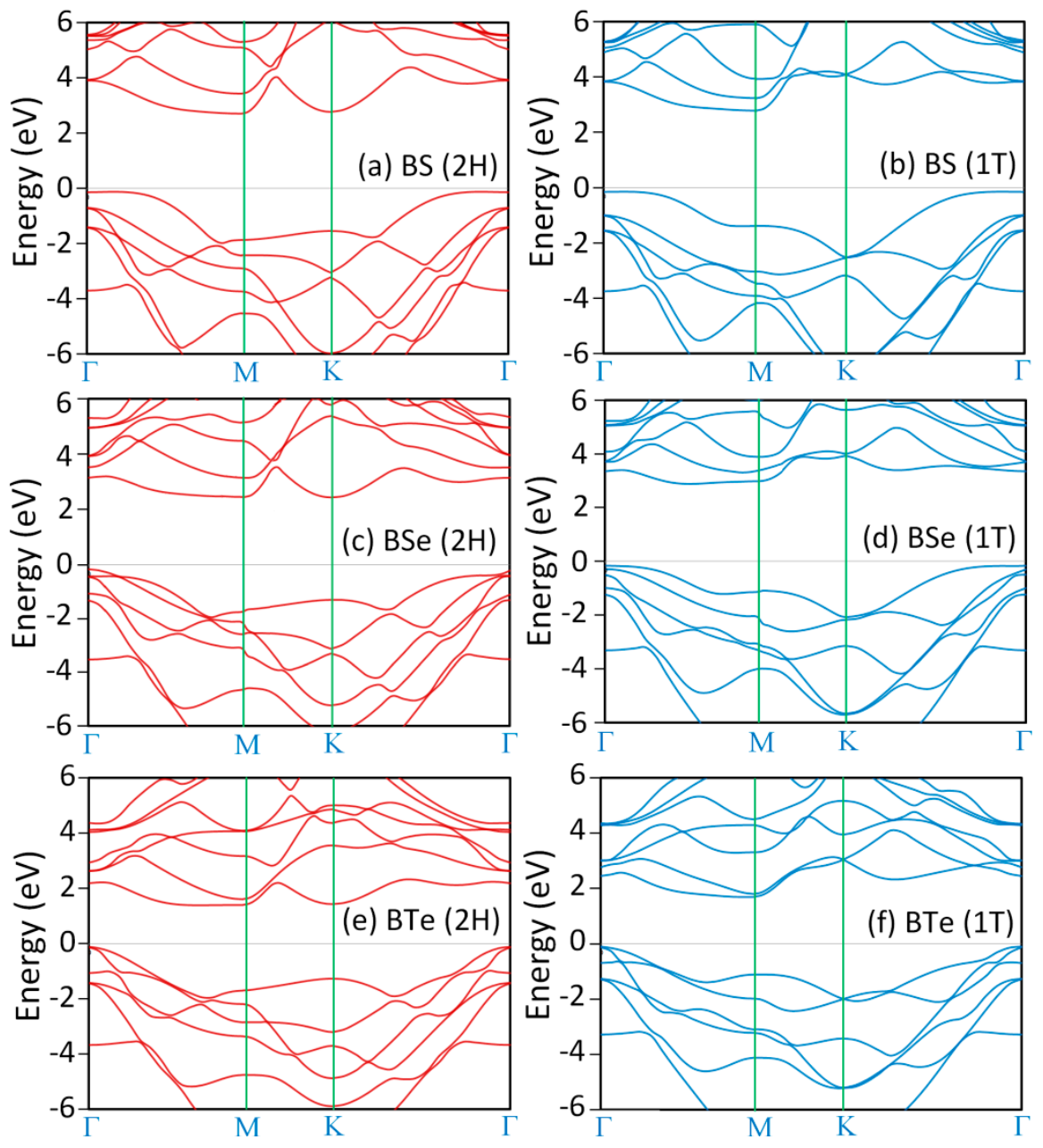

Figure 4. Band structure of single-layer BS, BSe, and BTe with $2 \mathrm{H}$ and $1 \mathrm{~T}$ atomic lattices predicted by the PBE functional. The Fermi energy is aligned to zero.

Since the PBE method underestimates the band gap, we employed the HSE06 functional to more accurately report the band gap values in these 2D systems. For 2H-BS, -BSe, and -BTe, the band gaps were measured to be $3.85 \mathrm{eV}, 2.96 \mathrm{eV}$, and $2.07 \mathrm{eV}$, respectively. In accordance with PBE results, the band gap of $1 \mathrm{~T}$ lattices were found to be slightly higher, $4.0 \mathrm{eV}, 3.43 \mathrm{eV}$, and $2.45 \mathrm{eV}$ for single-layer $\mathrm{BS}, \mathrm{BSe}$, and BTe, respectively. As is clear, BS and BTe monolayers exhibit the largest and narrowest band-gaps, respectively.

Mechanical properties are among the most critical properties of any material for the design of nanodevices [44-54]. We finally study the mechanical properties of boron monochalcogenides predicted in this work. In Figure 5, the DFT predictions for the uniaxial stress-strain responses of single-layer BS, BSe, and BTe with $2 \mathrm{H}$ and $1 \mathrm{~T}$ atomic lattices elongated along the armchair and zigzag directions are compared. For all of the predicted monolayers, the stress-strain response starts with an initial linear relation which is followed by a nonlinear trend up to the ultimate tensile strength point. The slope of the initial linear section of stress-strain curve is equal to the elastic modulus. In this work in order to report the elastic modulus, we fitted linear lines to the stress-strain values for the strain levels below 0.01 . Within the elastic region, the strain along the traverse direction $\left(\varepsilon_{\mathfrak{t}}\right)$ with respect to the loading strain $\left(\varepsilon_{1}\right)$ is constant and can be used to evaluate the Poisson's ratio, using: $-\varepsilon_{t} / \varepsilon_{1}$. In Table 2 , the mechanical properties of these 2D structures are summarized. Our results for the uniaxial loading along the armchair and zigzag directions show that linear sections coincide closely. As is also shown in Table 2, the elastic modulus along both considered loading directions are close, which suggest 
convincingly isotropic elasticity in the predicted BX monolayers. In contrast, the nonlinear sections of stress-strain responses are different depending on the loading direction. Interestingly, along the zigzag direction these systems show considerably higher tensile strength and stretchability as well. As is clear, the mechanical properties decline from BS to BTe. For the all BS monolayers, $2 \mathrm{H}$ lattices are stronger than $1 \mathrm{~T}$ phases. Interestingly, the strain at ultimate tensile strength which is the representative of the stretchability, is very high for BS and BTe monolayers with $2 \mathrm{H}$ lattice for the loading along the zigzag direction. Notably, along the zigzag direction the strain at ultimate tensile strength of $2 \mathrm{H}-\mathrm{Bs}$ and -BTe monolayers were found to be 0.26 and 0.3 , respectively, comparable to that of the pristine graphene ( 0.27 [55]) and hexagonal boron-nitride ( 0.3 [56]).
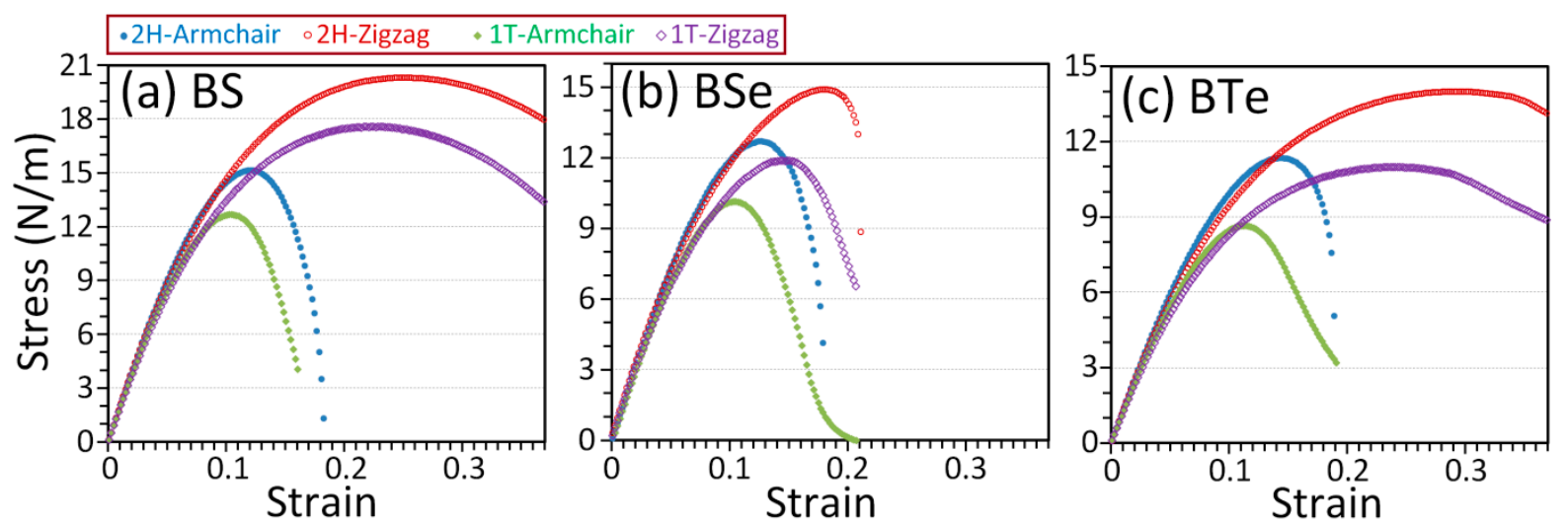

Figure 5. Uniaxial stress-strain responses of single-layer and free-standing boron monochalcogenides with $2 \mathrm{H}$ and $1 \mathrm{~T}$ phases stretched along the armchair and zigzag directions.

Table 2. Mechanical properties of single-layer BS, BSe and BTe with $2 \mathrm{H}$ and $1 \mathrm{~T}$ atomic lattices along the armchair and zigzag directions. $Y, P$, UTS and SUTS stand for elastic modulus, Poisson's ratio, ultimate tensile strength, and strain at ultimate tensile strength point, respectively. Stress units are in N/m.

\begin{tabular}{cccccccccc}
\hline \multicolumn{2}{c}{ Structure } & $\boldsymbol{Y}_{\text {armchair }}$ & $\boldsymbol{Y}_{\text {zigzag }}$ & $\boldsymbol{P}_{\text {armchair }}$ & $\boldsymbol{P}_{\text {zigzag }}$ & UTS $_{\text {armchair }}$ & UTS $_{\text {zigzag }}$ & SUTS $_{\text {armchair }}$ & SUTS $_{\text {zigzag }}$ \\
\hline \multirow{2}{*}{ BS } & 2H & 203 & 201 & 0.13 & 0.12 & 15.17 & 20.31 & 0.12 & 0.26 \\
& 1T & 195 & 193 & 0.13 & 0.12 & 12.69 & 17.60 & 0.11 & 0.23 \\
\multirow{2}{*}{ BSe } & 2H & 162 & 159 & 0.17 & 0.14 & 12.72 & 14.92 & 0.13 & 0.18 \\
& 1T & 155 & 155 & 0.17 & 0.14 & 10.15 & 11.89 & 0.11 & 0.15 \\
\multirow{2}{*}{ BTe } & 2H & 131 & 130 & 0.17 & 0.14 & 11.36 & 14.01 & 0.15 & 0.3 \\
& 1T & 122 & 121 & 0.17 & 0.14 & 11.00 & 8.66 & 0.12 & 0.27 \\
\hline
\end{tabular}

\section{Conclusions}

In this work we introduced boron monochalcogenides as a class of 2D materials. Two different atomic lattices for BS, BSe, and BTe monolayers with stacking sequences of ABBA and ABBC were considered. According to first-principles density functional theory simulation results, all predicted monolayers were found to be dynamically stable. Ab initio molecular dynamics simulations reveal the remarkable thermal stability of all predicted monolayers at high temperatures over $1500 \mathrm{~K}$. Electronic band structures confirm that all predicted single-layer boron monochalcogenides are indirect band gap semiconductors. According to the HSE06 method results, the band gaps of BS, BSe, and BTe monolayers with $2 \mathrm{H}$ atomic lattices were found to be $3.85 \mathrm{eV}, 2.96 \mathrm{eV}$, and $2.07 \mathrm{eV}$, respectively. In this regard, the electronic band-gap of $1 \mathrm{~T}$ lattices were found to be slightly higher, $4.0 \mathrm{eV}, 3.43 \mathrm{eV}$, and $2.45 \mathrm{eV}$ for single-layer BS, BSe, and BTe, respectively. Our first-principles results confirm convincingly isotropic elasticity and highly anisotropic tensile strengths in $2 \mathrm{H}$ and $1 \mathrm{~T}$ boron monochalcogenide nanomembranes. It was found that along the zigzag direction these nanosheets show considerably higher tensile strength and stretchability as well. As an interesting finding, the stretchability of BS 
and BTe nanomembranes along the zigzag were found to be comparable to that of the graphene and hexagonal boron-nitride. This study introduces a class of stable and strong 2D materials with inherent semiconducting electronic characters and, thus, we are hopeful that the obtained result can motivate future experimental and theoretical studies to explore the possible synthesis routes and new physics and chemistry of these nanomembranes.

Author Contributions: B.M. initiated the project, conducted the all simulations and wrote the manuscript under the supervision of T.R.

Acknowledgments: Authors greatly acknowledge the financial support by European Research Council for COMBAT project (grant number 615132).

Conflicts of Interest: The authors declare no conflict of interest.

\section{References}

1. Novoselov, K.S.; Geim, A.K.; Morozov, S.V.; Jiang, D.A.; Zhang, Y.; Dubonos, S.V.; Grigorieva, I.V.; Firsov, A.A. Electric field effect in atomically thin carbon films. Science 2004, 306, 666-669. [CrossRef] [PubMed]

2. Geim, A.K.; Novoselov, K.S. The rise of graphene. Nat. Mater. 2007, 6, 183-191. [CrossRef] [PubMed]

3. Novoselov, K.S.; Mishchenko, A.; Carvalho, A.; Neto, A.H.C. 2D materials and van der Waals heterostructures. Science 2016, 353, aac9439. [CrossRef] [PubMed]

4. Mounet, N.; Gibertini, M.; Schwaller, P.; Campi, D.; Merkys, A.; Marrazzo, A.; Sohier, T.; Castelli, I.E.; Cepellotti, A.; Pizzi, G.; et al. Two-dimensional materials from high-throughput computational exfoliation of experimentally known compounds. Nat. Nanotechnol. 2018, 13, 246-252. [CrossRef] [PubMed]

5. Oganov, A.R.; Glass, C.W. Crystal structure prediction using ab initio evolutionary techniques: Principles and applications. J. Chem. Phys. 2006, 124, 244704. [CrossRef] [PubMed]

6. Glass, C.W.; Oganov, A.R.; Hansen, N. USPEX-Evolutionary crystal structure prediction. Comput. Phys. Commun. 2006, 175, 713-720. [CrossRef]

7. Oganov, A.R.; Lyakhov, A.O.; Valle, M. How evolutionary crystal structure prediction works-and why. Acc. Chem. Res. 2011, 44, 227-237. [CrossRef] [PubMed]

8. Mannix, A.J.; Zhou, X.F.; Kiraly, B.; Wood, J.D.; Alducin, D.; Myers, B.D.; Liu, X.; Fisher, B.L.; Santiago, U.; Guest, J.R.; et al. Synthesis of borophenes: Anisotropic, two-dimensional boron polymorphs. Science 2015, 350, 1513-1516. [CrossRef] [PubMed]

9. Feng, B.; Zhang, J.; Zhong, Q.; Li, W.; Li, S.; Li, H.; Cheng, P.; Meng, S.; Chen, L.; Wu, K. Experimental Realization of Two-Dimensional Boron Sheets. Nat. Chem. 2016, 8, 563-568. [CrossRef] [PubMed]

10. Zhou, X.F.; Dong, X.; Oganov, A.R.; Zhu, Q.; Tian, Y.; Wang, H.T. Semimetallic two-dimensional boron allotrope with massless Dirac fermions. Phys. Rev. Lett. 2014, 112, 085502. [CrossRef]

11. Zhang, Z.; Yang, Y.; Gao, G.; Yakobson, B.I. Two-Dimensional Boron Monolayers Mediated by Metal Substrates. Angew. Chem. Int. Ed. 2015, 127, 13214-13218. [CrossRef]

12. Kubota, Y.; Watanabe, K.; Tsuda, O.; Taniguchi, T. Deep ultraviolet light-emitting hexagonal boron nitride synthesized at atmospheric pressure. Science 2007, 317, 932-934. [CrossRef] [PubMed]

13. Song, L.; Ci, L.; Lu, H.; Sorokin, P.B.; Jin, C.; Ni, J.; Kvashnin, A.G.; Kvashnin, D.G.; Lou, J.; Yakobson, B.I.; et al. Large scale growth and characterization of atomic hexagonal boron nitride layers. Nano Lett. 2010, 10, 3209-3215. [CrossRef] [PubMed]

14. Mahmood, J.; Lee, E.K.; Jung, M.; Shin, D.; Choi, H.J; Seo, J.M.; Jung, S.M.; Kim, D.; Li, F.; Lah, M.S.; et al. Two-dimensional polyaniline (C3N) from carbonized organic single crystals in solid state. Proc. Natl. Acad. Sci. USA 2016, 113, 7414-7419. [CrossRef] [PubMed]

15. Mahmood, J.; Lee, E.K.; Jung, M.; Shin, D.; Jeon, I.Y.; Jung, S.M.; Choi, H.J.; Seo, J.M.; Bae, S.Y.; Sohn, S.D.; et al. Nitrogenated holey two-dimensional structures. Nat. Commun. 2015, 6, 6486. [CrossRef] [PubMed]

16. Thomas, A.; Fischer, A.; Goettmann, F.; Antonietti, M.; Müller, J.O.; Schlögl, R.; Carlsson, J.M. Graphitic carbon nitride materials: Variation of structure and morphology and their use as metal-free catalysts. J. Mater. Chem. 2008, 18, 4893-4908. [CrossRef]

17. Algara-Siller, G.; Severin, N.; Chong, S.Y.; Björkman, T.; Palgrave, R.G.; Laybourn, A.; Antonietti, M.; Khimyak, Y.Z.; Krasheninnikov, A.V.; Rabe, J.P.; et al. Triazine-based graphitic carbon nitride: A two-dimensional semiconductor. Angew. Chem. 2014, 53, 7450-7455. [CrossRef] [PubMed] 
18. Aufray, B.; Kara, A.; Vizzini, S.; Oughaddou, H.; Leandri, C.; Ealet, B.; Le Lay, G. Graphene-like silicon nanoribbons on $\mathrm{Ag}(110)$ : A possible formation of silicene. Appl. Phys. Lett. 2010, 96, 183102. [CrossRef]

19. Vogt, P.; De Padova, P.; Quaresima, C.; Avila, J.; Frantzeskakis, E.; Asensio, M.C.; Resta, A.; Ealet, B.; Le Lay, G. Silicene: Compelling experimental evidence for graphenelike two-dimensional silicon. Phys. Rev. Lett. 2012, 108, 155501. [CrossRef] [PubMed]

20. Bianco, E.; Butler, S.; Jiang, S.; Restrepo, O.D.; Windl, W.; Goldberger, E.J. Stability and exfoliation of germanane: A germanium graphane analogue. ACS Nano 2013, 7, 4414-4421. [CrossRef] [PubMed]

21. Geim, A.K.; Grigorieva, I.V. Van der Waals heterostructures. Nature 2013, 499, 419-425. [CrossRef] [PubMed]

22. Wang, Q.H.; Kalantar-Zadeh, K.; Kis, A.; Coleman, J.N.; Strano, M.S. Electronics and optoelectronics of two-dimensional transition metal dichalcogenides. Nat. Nanotechnol. 2012, 7, 699-712. [CrossRef] [PubMed]

23. Radisavljevic, B.; Radenovic, A.; Brivio, J.; Giacometti, V.; Kis, A. Single-layer MoS $_{2}$ transistors. Nat. Nanotechnol. 2011, 6, 147-150. [CrossRef] [PubMed]

24. Das, S.; Demarteau, M.; Roelofs, A. Ambipolar phosphorene field effect transistor. ACS Nano 2014, 8, 11730-11738. [CrossRef] [PubMed]

25. Li, L.; Yu, Y.; Ye, G.J.; Ge, Q.; Ou, X.; Wu, H.; Feng, D.; Chen, X.H.; Zhang, Y. Black phosphorus field-effect transistors. Nat. Nanotechnol. 2014, 9, 372-377. [CrossRef] [PubMed]

26. Bandurin, D.A.; Tyurnina, A.V.; Geliang, L.Y.; Mishchenko, A.; Zólyomi, V.; Morozov, S.V.; Kumar, R.K.; Gorbachev, R.V.; Kudrynskyi, Z.R.; Pezzini, S.; et al. High electron mobility, quantum Hall effect and anomalous optical response in atomically thin InSe. Nat. Nano 2017, 12, 223-227. [CrossRef] [PubMed]

27. Cherednichenko, K.A.; Kruglov, I.A.; Oganov, A.R.; le Godec, Y.; Mezouar, M.; Solozhenko, V.L. Boron monosulfide: Equation of state and pressure-induced phase transition. J. Appl. Phys. 2018, 123, 135903. [CrossRef]

28. Zólyomi, V.; Drummond, N.D.; Fal'Ko, V.I. Electrons and phonons in single layers of hexagonal indium chalcogenides from ab initio calculations. Phys. Rev. B Condens. Matter Mater. Phys. 2014, 89, 205416. [CrossRef]

29. Fan, D.; Yang, C.; Lu, S.; Hu, X. Two-Dimensional Boron Monosulfides: Semiconducting and Metallic Polymorphs. arXiv 2018, arXiv:1803.03459.

30. Kresse, G. From ultrasoft pseudopotentials to the projector augmented-wave method. Phys. Rev. B 1999, 59, 1758-1775. [CrossRef]

31. Kresse, G.; Furthmüller, J. Efficiency of ab-initio total energy calculations for metals and semiconductors using a plane-wave basis set. Comput. Mater. Sci. 1996, 6, 15-50. [CrossRef]

32. Kresse, G.; Furthmüller, J. Efficient iterative schemes for ab initio total-energy calculations using a plane-wave basis set. Phys. Rev. B 1996, 54, 11169-11186. [CrossRef]

33. Perdew, J.; Burke, K.; Ernzerhof, M. Generalized Gradient Approximation Made Simple. Phys. Rev. Lett. 1996, 77, 3865-3868. [CrossRef] [PubMed]

34. Blöchl, P.E.; Jepsen, O.; Andersen, O.K. Improved tetrahedron method for Brillouin-zone integrations. Phys. Rev. B 1994, 49, 16223-16233. [CrossRef]

35. Monkhorst, H.; Pack, J. Special points for Brillouin zone integrations. Phys. Rev. B 1976, 13, 5188-5192. [CrossRef]

36. Krukau, A.V.; Vydrov, O.A.; Izmaylov, A.F.; Scuseria, G.E. Influence of the exchange screening parameter on the performance of screened hybrid functionals. J. Chem. Phys. 2006, 125, 224106. [CrossRef] [PubMed]

37. Togo, A.; Oba, F.; Tanaka, I. First-principles calculations of the ferroelastic transition between rutile-type and $\mathrm{CaCl}_{2}$-type $\mathrm{SiO}_{2}$ at high pressures. Phys. Rev. B Condens. Matter Mater. Phys. 2008, 78, 134106. [CrossRef]

38. Lin, Y.-C.; Dumcenco, D.O.; Huang, Y.-S.; Suenaga, K. Atomic mechanism of the semiconducting-to-metallic phase transition in single-layered $\mathrm{MoS}_{2}$. Nat. Nanotechnol. 2014, 9, 391-396. [CrossRef] [PubMed]

39. Mattheiss, L.F. Band structures of transition-metal-dichalcogenide layer compounds. Phys. Rev. B 1973, 8, 3719-3740. [CrossRef]

40. Wypych, F.; Schöllhorn, R.; Schollhorn, R.; Schöllhorn, R. 1T-MoS 2 , a new metallic modification of molybdenum disulfide. J. Chem. Soc. Chem. Commun. 1992, 19, 1386-1388. [CrossRef]

41. Momma, K.; Izumi, F. VESTA 3 for three-dimensional visualization of crystal, volumetric and morphology data. J. Appl. Crystallogr. 2011, 44, 1272-1276. [CrossRef]

42. Silvi, B.; Savin, A. Classification of Chemical-Bonds Based on Topological Analysis of Electron Localization Functions. Nature 1994, 371, 683-686. [CrossRef] 
43. Henkelman, G.; Arnaldsson, A.; Jónsson, H. A fast and robust algorithm for Bader decomposition of charge density. Comput. Mater. Sci. 2006, 36, 354-360. [CrossRef]

44. Sadeghzadeh, S. Borophene sheets with in-plane chain-like boundaries; a reactive molecular dynamics study. Comput. Mater. Sci. 2018, 143, 1-14. [CrossRef]

45. Sadeghzadeh, $\mathrm{S}$. The creation of racks and nanopores creation in various allotropes of boron due to the mechanical loads. Superlattices Microstruct. 2017, 111, 1145-1161. [CrossRef]

46. Le, M.-Q.; Batra, R.C. Mode-I stress intensity factor in single layer graphene sheets. Comput. Mater. Sci. 2016, 118, 251-258. [CrossRef]

47. Ganji, A.R.P.; Armat, M.R.; Tabatabaeichehr, M.; Mortazavi, H. The Effect of Self-Management Educational Program on Pain Intensity in Elderly Patients with Knee Osteoarthritis: A Randomized Clinical Trial. Open Access Maced. J. Med. Sci. 2018. [CrossRef]

48. Mortazavi, H. Designing a multidimensional pain assessment tool for critically Ill elderly patients: An agenda for future research. Indian J. Crit. Care Med. 2018, 22, 390-391. [CrossRef]

49. Le, M.-Q. Reactive molecular dynamics simulations of the mechanical properties of various phosphorene allotropes. Nanotechnology 2018, 29, 195701. [CrossRef] [PubMed]

50. Nguyen, D.T.; Le, M.Q.; Nguyen, V.T.; Bui, T.L. Effects of various defects on the mechanical properties of black phosphorene. Superlattices Microstruct. 2017, 112, 186-199. [CrossRef]

51. Shirazi, A.H.N.; Abadi, R.; Izadifar, M.; Alajlan, N.; Rabczuk, T. Mechanical responses of pristine and defective C3N nanosheets studied by molecular dynamics simulations. Comput. Mater. Sci. 2018, 147, 316-321. [CrossRef]

52. Abadi, R.; Shirazi, A.H.N.; Izadifar, M.; Sepahi, M.; Rabczuk, T. Fabrication of nanopores in polycrystalline boron-nitride nanosheet by using $\mathrm{Si}, \mathrm{SiC}$ and diamond clusters bombardment. Comput. Mater. Sci. 2018, 145, 280-290. [CrossRef]

53. Shi, L.B.; Zhang, Y.Y.; Xiu, X.M.; Dong, H.K. Structural Characteristics and Strain Behaviors of Two-Dimensional $C_{3}$ N: First Principles Calculations. Carbon 2018, 134, 103-111. [CrossRef]

54. Mortazavi, H.; Tabatabaeichehr, M.; Taherpour, M.; Masoumi, M. Relationship Between Home Safety and Prevalence of Falls and Fear of Falling Among Elderly People: A Cross-Sectional Study. Mater. Soc. Med. 2018, 30, 103-107. [CrossRef]

55. Liu, F.; Ming, P.; Li, J. Ab initio calculation of ideal strength and phonon instability of graphene under tension. Phys. Rev. B Condens. Matter Mater. Phys. 2007, 76, 064120. [CrossRef]

56. Peng, Q.; Ji, W.; De, S. Mechanical properties of the hexagonal boron nitride monolayer: Ab initio study. Comput. Mater. Sci. 2012, 56, 11-17. [CrossRef] 